\title{
Long waves over a bi-viscous seabed: transverse patterns
}

\author{
J. M. Becker ${ }^{1}$ and D. Bercovici ${ }^{1,}$ * \\ ${ }^{1}$ Department of Geology and Geophysics, University of Hawaii at Manoa, Honolulu, HI, USA \\ *Present address Department of Geology and Geophysics, Yale University, New Haven, CT, USA
}

Received: 10 October 2000 - Revised: 2 July 2001 - Accepted: 3 September 2001

\begin{abstract}
The coupled interaction of long standing hydrodynamic waves with a deformable non-Newtonian seabed is examined using a two-layer model for which the upper layer fluid is inviscid and the lower layer is bi-viscous. The two-dimensional response of the system to forcing by a predominantly longitudinal (cross-shore) standing wave perturbed by a small transverse (along-shore) component is determined. With a constant yield stress in the bi-viscous lower layer, there is little amplification of these transverse perturbations and the model response typically remains quasione-dimensional. However, for a bi-viscous layer with a pressure-dependent yield stress (which represents the effect that the seabed deforms less readily under compression and hence renders the rheology history dependent), the initially small transverse motions are amplified in some parameter regimes and two-dimensional, permanent bedforms are formed in the lower layer. This simple dynamical model is, therefore, able to explain the formation of permanent bedforms with significant cross- and along-shore features by predominantly cross- shore standing wave forcing.
\end{abstract}

\section{Introduction}

The interaction of ocean waves and beaches forms a highly complex system and observations reveal that pattern formation occurs in the nearshore. These regular morphological features have spatial scales that range from centimeters for sand ripples, to kilometers for sand bars, and often maintain their structure for time scales significantly longer than the ocean forcing (Plant et al., 1999). While a variety of modeling efforts have been carried out to gain insight into the interaction of waves and beaches, no consensus has yet been reached about how best to model the formation of nearshore morphology or the impact that the seabed morphology has on nearshore sediment transport (Thornton et al., 1998).

Correspondence to: J. M. Becker

(jbecker@soest.hawaii.edu)
Many models of pattern formation in the nearshore couple the dynamics for the overlying fluid motion to a continuity equation for the sediment by using a prescribed sediment flux law. For example, Blondeaux (1990) (see also Vittori and Blondeaux, 1990, 1992) examined the generation of smallscale sand ripples by sea waves by coupling the flow in the viscous oscillatory boundary layer to the continuity equation for the sediment using an empirically determined sediment flux relationship. Stability boundaries for various sand ripple patterns that are in reasonable agreement with observations were obtained; however, these results rely on the parameterization of the sediment flux that is not well understood.

The approach of coupling the dynamics of the fluid motion to the seabed using a sediment transport formula also has been applied in models of the formation of large-scale seabed morphology. This approach is the basis for many cross-shore sediment transport models (Roelvink and Broker, 1993) and also has been used in theoretical models of the formation of longshore sand bars (Carter et al., 1973; BoczarKarakiewicz, et al., 1995) and more complex morphologies (Holman and Bowen, 1982). In addition, a variety of new modeling efforts have been developed to explain pattern formation in the nearshore. These studies also are based upon hydrodynamics coupled to sediment transport formulas, but in contrast to the aforementioned studies, use a linear stability analysis to determine instabilities in the coupled waveseabed system. For example, Falques et al. (1996) considered the stability of a longshore current over a plane erodible bed and found instabilities that correspond to the formation of longshore periodic beach topographies. Deigaard et al. (1999) considered the stability of waves and a longshore current over a barred erodible bed and found instabilities that correspond to rip channels. In an earlier study, a rule-based model that uses a kinematical description of the swash flow and a prescribed sediment flux law was developed to describe the formation of beach cusps (Werner and Fink, 1993).

Here, we follow the suggestion by Holman (1995) that a promising way to gain new insights into the evolution of nearshore morphology is to consider simplified models 
that capture the essential nonlinear dynamics of the coupled ocean-seabed system. We recently have demonstrated that a simple nonlinear dynamical model of the coupled interaction of waves with a deformable seabed can describe the formation of small wavelength bedforms that maintain their structure over many periods of forcing (Becker and Bercovici, 2000). Our model is based upon the hypothesis that sand may be modeled as a continuum with a non-Newtonian rheology. With a non-Newtonian rheology, it is possible to approximate important features of sand motion such as critical bed shear stress (i.e. the sand bed will deform given a sufficiently large stress, but will retain a solid structure otherwise) and history-dependent loading (i.e. sand is more difficult to move under loading than unloading). We remark that nonNewtonian rheologies have been used previously to model mud flows and muddy seabeds (Liu and Mei, 1989, 1993a, b; Ng and Mei, 1994).

The interaction of waves with a non-Newtonian seabed first was examined by Liu and Mei (1993a, b) who considered a two-layer model in the long-wave limit for which the upper layer was inviscid and the lower layer was a Bingham plastic. They showed that transient waves generated by an initial free-surface displacement leave behind permanent "foot-prints" in the Bingham plastic layer. In the aforementioned study of Becker and Bercovici (2000), the two-layer model of Liu and Mei (1993a, b) was revisited to determine whether or not persistent forcing by standing waves would generate permanent features in the non-Newtonian seabed modeled as a bi-viscous fluid (the Bingham rheology is a limiting form of the bi-viscous rheology) and how the spatial scale of the lower layer pattern was related to that of the standing wave forcing. By forcing the two-layer system with an external surface pressure, Becker and Bercovici (2000) showed that the nonlinear rheology transferred energy from the forced wave motion to odd spatial harmonics in the lower layer, as is consistent with the symmetry of the bi-viscous rheology (i.e. since the stress is an odd function of the strain rate). These lower layer features, however, oscillated with the forcing frequency; hence, permanent bedforms did not form under persistent standing wave forcing for a constant yield stress bi-viscous lower layer. By considering a more realistic (although still highly idealized rheology) for the lower layer that accounted for the observation that sand deforms less readily under compression, Becker and Bercovici (2000) showed that energy may be transferred to even and odd harmonics of the forcing and that permanent bedforms with spatial scales half that of the forcing were formed in the lower layer. We remark that in Liu and Mei (1993a, b), the permanent footprint in the Bingham plastic lower layer remains after the passing of the transient water wave. In the present study, the permanent bedforms in the bi-viscous lower layer are interfacial patterns with a non-zero time average over a period of the oscillatory forcing.

The studies of Liu and Mei (1993a, b) and Becker and Bercovici (2000) were developed in an effort to understand the cross-shore dependence of the interaction of seawaves with the non-Newtonian seabed and hence were one- dimensional. Observations reveal, however, that bedforms with significant cross- and along-shore dependence may form (e.g. Vittori and Blondeaux, 1992; Holman and Bowan, 1982). Here, we examine the two-dimensional model dynamics to determine under what conditions predominately cross-shore (longitudinal) forcing may generate bedforms with significant along-shore (transverse) structure. As in Becker and Bercovici (2000), we excite standing waves by forcing the system with a harmonic external surface pressure of magnitude $A$, but here allow for small transverse pressure perturbations of magnitude $A^{\prime}$, where $A^{\prime} \ll A$ :

$P_{0}=A \cos (k x) \sin (\omega t)+A^{\prime} \cos (l y) \sin (\sigma t)$

and $(k, \omega)$ and $(l, \sigma)$ are the wave number and frequency pairs of the longitudinal pressure forcing and transverse perturbation, respectively. In the limit of a constant yield stress bi-viscous lower layer, the transverse pressure perturbations typically remain $O\left(A^{\prime}\right)$ and as a result, the spatial pattern formed in the lower layer is quasi-one-dimensional (with one notable exception, see Sect. 3). In addition, in all cases examined for the constant yield stress bi-viscous lower layer, these interfacial patterns oscillate with the forcing frequency and hence are not permanent. In contrast, for a bi-viscous lower layer with a pressure-dependent yield stress, the transverse perturbations are amplified for a range of forcing parameters. Moreover, the two-dimensional spatial patterns in the interface displacement that are excited have a significant non-zero time average and result in the formation of permanent bedforms with significant along-shore structure.

\section{Dynamics}

We consider here two superposed shallow homogeneous layers of fluid (Fig. 1) with equilibrium layer depths $H_{1}$ and $H_{2}$, free-surface displacement $\zeta_{1}(\mathbf{x}, t)$ and interface displacement $\zeta_{2}(\mathbf{x}, t)$, where $\mathbf{x}=(x, y)$. Both the upper and lower layer fluids are incompressible with constant densities $\rho_{j} \quad(j=$ $1,2)$, and the upper layer fluid is inviscid, while the lower layer fluid is bi-viscous.

The long-wave momentum equations for this two-layer system in the absence of inertia are

$$
\begin{aligned}
& \frac{\partial \mathbf{u}_{1}}{\partial t}=-g \nabla \zeta_{1}-\frac{1}{\rho_{1}} \nabla P_{0} \\
& \frac{\partial \overline{\mathbf{u}}_{2}}{\partial t}=-g \nabla\left(\zeta_{2}+\frac{\rho_{1}}{\rho_{2}}\left[\zeta_{1}-\zeta_{2}\right]\right)-\frac{1}{\rho_{2}} \nabla P_{0}-\frac{\boldsymbol{\tau}_{b}}{\rho_{2} H_{2}}
\end{aligned}
$$

and the linearized continuity equations are

$$
\begin{aligned}
& \frac{\partial\left(\zeta_{1}-\zeta_{2}\right)}{\partial t}+H_{1} \nabla \cdot \mathbf{u}_{1}=0 \\
& \frac{\partial \zeta_{2}}{\partial t}+H_{2} \nabla \cdot \overline{\mathbf{u}}_{2}=0
\end{aligned}
$$

where $\mathbf{u}_{1}$ is the horizontal fluid velocity in the inviscid upper layer, $\overline{\mathbf{u}}_{2}$ is the depth-averaged horizontal fluid velocity in 


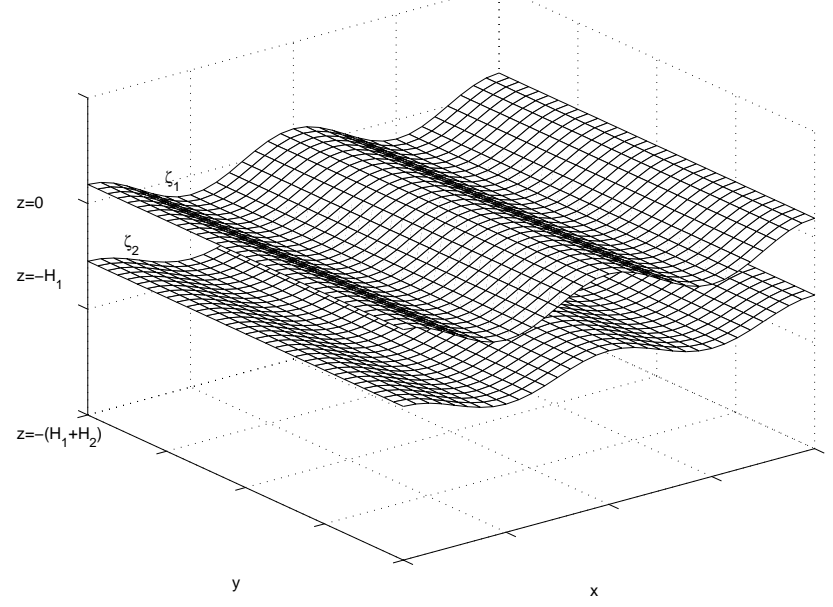

Fig. 1. The two-layer model. The upper layer fluid is inviscid and incompressible, and is confined to $\zeta_{2}-H_{1}<z<\zeta_{1}$. The lower layer fluid is bi-viscous (see Eq. 6) and incompressible, and is confined to $-\left(H_{1}+H_{2}\right)<z<\zeta_{2}-H_{1}$.

the lower layer, $\nabla=\left(\partial_{x}, \partial_{y}\right)$ is the horizontal gradient operator, $g$ is gravitational acceleration, $\boldsymbol{\tau}_{b}=\left.\left(\tau_{x z}, \tau_{y z}\right)\right|_{z=-H}$ is the bottom stress and $P_{0}(\mathbf{x}, t)$ is the applied surface pressure given in Eq. (1). A derivation of the one-dimensional versions of Eqs. (2)-(5) may be found in Becker and Bercovici (2001).

The dependence of the stress on the strain rate warrants some motivation which is provided below. We emphasize, however, that this lower layer rheology is highly idealized and is not meant to represent a unique continuum model of sand and sediment motion.

\subsection{The lower layer rheology}

We first consider a bi-viscous rheology for the lower layer because it is perhaps the simplest model of the two flow states of a sediment laden seabed; the seabed resists motion for small applied stresses, but flows readily for stresses above a critical value. For a bi-viscous lower layer, the stress $\boldsymbol{\tau}=\left(\tau_{x z}, \tau_{y z}\right)$ is related to the long-wave strain rate $\dot{\boldsymbol{\varepsilon}}=\left(\dot{\varepsilon}_{x z}, \dot{\varepsilon}_{y z}\right)=\frac{\partial \mathbf{u}_{2}}{\partial z}\left(\mathbf{u}_{2}\right.$ is the $z$-dependent lower layer velocity) according to

$$
\begin{aligned}
& \frac{1}{\mu} \boldsymbol{\tau}=\dot{\boldsymbol{\varepsilon}}, \quad \hat{\tau} \leq \tau_{0 p} \\
& \frac{1}{\mu^{\prime}} \boldsymbol{\tau}=\tau_{0 p}\left(\frac{1}{\mu^{\prime}}-\frac{1}{\mu}\right) \frac{\dot{\boldsymbol{\varepsilon}}}{\hat{\dot{\varepsilon}}}+\dot{\boldsymbol{\varepsilon}}, \quad \hat{\tau}>\tau_{0 p} .
\end{aligned}
$$

In Eq. (6), $\tau_{0 p}, \mu$ and $\mu^{\prime}$ are the yield stress and dynamic viscosities respectively, $\hat{\tau}=\left(\tau_{x z}^{2}+\tau_{y z}^{2}\right)^{1 / 2}$ and $\hat{\dot{\varepsilon}}=\left(\dot{\varepsilon}_{x z}^{2}+\dot{\varepsilon}_{y z}^{2}\right)^{1 / 2}$. Equation (6) describes a bi-viscous rheology with a yield stress, $\tau_{0 p}$, that may depend upon space and time. The Newtonian rheology may be recovered from Eq. (6) in the limit as $\mu^{\prime} \rightarrow \mu$ (or equivalently $\tau_{0 p} \rightarrow \infty$ ), while the Bingham rheology may be recovered from Eq. (6) for a constant yield stress in the limit as $\mu$ becomes infinite.
The lower layer rheology, Eq. (6), relates the stress to the strain rate for all values of the vertical coordinate. Since only the bottom stress $\boldsymbol{\tau}_{b}$ occurs in the depth-averaged lower layer momentum balance, Eq. (3), we approximate the strain rate at $z=-H$ in terms of the average lower layer velocity according to

$\left.\dot{\boldsymbol{\varepsilon}}_{b} \equiv \frac{\partial \mathbf{u}_{2}}{\partial z}\right|_{z=-H} \approx \frac{\overline{\mathbf{u}}_{2}}{H_{2}}$.

We remark that the approximation Eq. (7) differs by an $O(1)$ factor from the bottom strain rate obtained for a Newtonian lower layer in the limit of creeping flow.

The dynamics of Eqs. (1)-(7) govern the evolution of the free-surface and interface displacements, and the upper and lower layer horizontal fluid velocities. To close this set of equations, we specify next the yield stress $\tau_{0 p}$.

In what follows, we examine two rheologies for the lower layer. In the first and simplest case, the yield stress is a constant $\tau_{0}\left(\tau_{0 p}=\tau_{0}\right)$ and Eq. (6) reduces to the bi-viscous rheology considered by Liu and Mei (1993b). In this case, when $\mu \gg \mu^{\prime}$, the lower layer dynamic balance for small applied stresses with $\tau_{b} \leq \tau_{0}$ is creeping flow, while for larger applied stresses with $\tau_{b}>\tau_{0}$, the dynamics are those of weakly-damped internal waves (Becker and Bercovici, 2001). We emphasize, however, that the bottom stress is a function of space and time; hence, at a particular time, the viscosity of the lower layer fluid may vary in space depending upon the local value of the bottom stress.

The second rheology considered here models the observation that a seabed yields less readily under compression than extension. As a result, we allow the yield stress, $\tau_{0 p}$, to be a simple linear function of the dynamic bottom pressure according to:

$$
\begin{gathered}
\tau_{0 p}=\tau_{0}\left(1+C p_{b}(\mathbf{x}, t)\right), \quad 0 \leq C<C_{\text {max }} \\
p_{b}=\rho_{2} g \zeta_{2}+\rho_{1} g\left(\zeta_{1}-\zeta_{2}\right)+P_{0} .
\end{gathered}
$$

When $C=0$, we recover the constant yield stress bi-viscous rheology described above. For $0<C<C_{\max }$, Eq. (8) represents a Coulomb-type friction, so that the yield stress depends upon the normal stress. In regions where the normal stress (bottom pressure) is positive/negative, the yield stress is elevated/depressed. Hence, this pressure-dependent yield stress represents a packing condition: for a positive normal stress, the bed resists motion for a larger range of stresses, since the yield stress is elevated (representing the relative difficulty of setting compacted grains in motion), while for a negative normal stress, the bed is more easily eroded because the yield stress is depressed (representing the relative ease of setting loosely packed grains in motion). In Eq. (8), $C$ is a constant that varies from 0 for a constant yield stress lower layer to an empirically determined maximum value $C_{\text {max }}$ chosen so that $\tau_{0 p}$ undergoes significant variations, but remains positive. Similar results to those reported in Sect. 3 also have been obtained with a yield stress that is an exponential function of the bottom pressure given by

$\tau_{0 p}=\tau_{0} e^{C p_{b}(\mathbf{x}, t)}$ 
so that $\tau_{0 p}$ is positive for all values of $C$; obviously, Eq. (8) is the first two terms in a Taylor expansion of Eq. (9). Finally, we remark that we have neglected fluid inertia in Eqs. (2)-(3) and linearized the continuity Eqs. (4)-(5) to isolate the effects of nonlinearity due to the lower layer rheology Eqs. (6)-(8); hence, the model dynamics are valid in the small-amplitude limit.

\subsection{Non-dimensional dynamics}

We non-dimensionalize Eqs. (1)-(8) according to

$$
\begin{aligned}
& (\mathbf{x}, t)=L\left(\mathbf{x}^{*}, c^{-1} t^{*}\right) \\
& \left(\mathbf{u}_{\mathbf{j}}, \zeta_{j}\right)=\frac{A k L}{\rho_{1} c^{2}}\left(c \mathbf{u}_{\mathbf{j}}^{*}, H \zeta_{j}^{*}\right) \quad(j=1,2)
\end{aligned}
$$

and

$$
P_{0}=A \Pi_{0}, \quad\left(\boldsymbol{\tau}, \tau_{0 p}\right)=A k H \frac{\rho_{2}}{\rho_{1}}\left(\Upsilon, \Upsilon_{0 p}\right),
$$

where the ${ }^{*}$ superscript indicates dimensionless quantities, $\Pi_{0}, \Upsilon$, and $\Upsilon_{0 p}$ are the dimensionless applied pressure, stress, and yield stress respectively, $L$ is a representative horizontal lengthscale and $c=(g H)^{1 / 2}$.

As mentioned in the Introduction, we excite waves in the system with the (dimensionless) applied surface pressure

$\Pi_{0}=\cos \left(\kappa x^{*}\right) \sin \left(\Omega t^{*}\right)+\alpha \cos \left(\lambda y^{*}\right) \sin \left(\Sigma t^{*}\right)$,

where $\alpha=\frac{A^{\prime}}{A} \ll 1$ is the ratio of the magnitude of the pressure perturbation to the pressure forcing, and $(\kappa, \lambda)=L(k, l)$ are the nondimensional wave numbers and $(\Omega, \Sigma)=\frac{L}{c}(\omega, \sigma)$ are the nondimensional frequencies of the pressure forcing and perturbation, respectively. We force standing waves by choosing $\kappa=m \pi$ and consider transverse standing wave perturbations with $\lambda=n \pi$ for a variety of $(n, m)$ where $n$ and $m$ are integers.

In dimensionless form, the governing equations become

$$
\begin{aligned}
& \frac{\partial \mathbf{u}_{1}^{*}}{\partial t^{*}}=-\nabla^{*} \zeta_{1}^{*}+\sin \left(m \pi x^{*}\right) \sin \left(\Omega t^{*}\right) \hat{\mathbf{i}} \\
& \quad+\alpha \sin \left(n \pi y^{*}\right) \sin \left(\Sigma t^{*}\right) \hat{\mathbf{j}}
\end{aligned}
$$

$$
\begin{aligned}
& \frac{\partial \overline{\mathbf{u}}_{2}^{*}}{\partial t^{*}}=-\nabla^{*}\left[\zeta_{2}^{*}+s\left(\zeta_{1}^{*}-\zeta_{2}^{*}\right)\right]-\frac{\Upsilon_{b}}{H_{2}^{*}} \\
& \quad+s \sin \left(m \pi x^{*}\right) \sin \left(\Omega t^{*}\right) \hat{\mathbf{i}} \\
& \quad+\alpha s \sin \left(n \pi y^{*}\right) \sin \left(\Sigma t^{*}\right) \hat{\mathbf{j}} \\
& \frac{\partial\left(\zeta_{1}^{*}-\zeta_{2}^{*}\right)}{\partial t^{*}}+H_{1}^{*} \nabla^{*} \cdot \mathbf{u}_{1}^{*}=0 \\
& \frac{\partial \zeta_{2}^{*}}{\partial t^{*}}+H_{2}^{*} \nabla^{*} \cdot \overline{\mathbf{u}}_{2}^{*}=0
\end{aligned}
$$

where $(\hat{\mathbf{i}}, \hat{\mathbf{j}})$ are unit vectors in the $(x, y)$ directions, $s=\frac{\rho_{1}}{\rho_{2}}$ is the density ratio, $\left(\Gamma, \Gamma^{\prime}\right)=\frac{L}{\rho_{2} c H^{2}}\left(\mu, \mu^{\prime}\right)$ are the nondimensional viscosity parameters and $\left(H_{1}^{*}, H_{2}^{*}\right)=H^{-1}\left(H_{1}, H_{2}\right)$ are the nondimensional layer depths. The nondimensional bottom stress $\Upsilon_{b}=\left.\Upsilon \Upsilon \Upsilon\right|_{z^{*}=-H^{*}}$ is given by

$$
\begin{aligned}
& \frac{1}{\Gamma} \Upsilon_{b}=\frac{\overline{\mathbf{u}}_{2}^{*}}{H_{2}^{*}}, \quad \hat{\Upsilon}_{b} \leq \Upsilon_{0 p} \\
& \frac{1}{\Gamma^{\prime}} \Upsilon_{b}=\frac{\Upsilon_{0 p}}{\hat{\dot{\varepsilon}}_{b}^{*}}\left(\frac{1}{\Gamma^{\prime}}-\frac{1}{\Gamma}\right) \frac{\overline{\mathbf{u}}_{2}^{*}}{H_{2}^{*}}+\frac{\overline{\mathbf{u}}_{2}^{*}}{H_{2}^{*}}, \quad \hat{\Upsilon}_{b}>\Upsilon_{0 p},
\end{aligned}
$$

where $\left(\hat{\Upsilon}_{b}, \hat{\dot{\varepsilon}}_{b}^{*}\right)$ are the magnitudes of the nondimensional bottom stress and strain rate, and

$$
\begin{gathered}
\Upsilon_{0 p}\left(\mathbf{x}^{*}, t^{*}\right)=\Upsilon_{0}\left(1+\mathcal{C} p_{b}^{*}\left(\mathbf{x}^{*}, t^{*}\right)\right), \quad 0 \leq \mathcal{C}<\mathcal{C}_{\max } \\
p_{b}^{*}\left(\mathbf{x}^{*}, t^{*}\right)=s\left(\Pi_{0}+\zeta_{1}^{*}-\zeta_{2}^{*}\right)+\zeta_{2}^{*}
\end{gathered}
$$

is the pressure-dependent yield stress, where $\mathcal{C}=0$ for a constant yield stress lower layer $\left(\Upsilon_{0 p}=\Upsilon_{0}\right)$, and $\mathcal{C} \neq 0$ for a lower layer with a Coulomb-type friction.

\section{Numerical solutions}

We solve Eqs. (11)-(16) numerically using a finite-difference method on a domain given by $0<x<2,0<y<2$ subject to the condition of no normal flow at the boundaries. We time-step the dependent variables using a centereddifference scheme supplemented with a forward time-step every 10 steps in order to to supress the computational mode. The time-step is chosen so that the CFL condition is satisfied. The number of grid points in space required to obtain a converged solution is dictated by resolving the highest wave number excited and hence depends upon the forcing wave numbers and frequencies in Eq. (10) (see below). We have benchmarked this code against the pseudo-spectral method of Becker and Bercovici (2000) and the analytic solution for a Newtonian lower layer derived in Becker and Bercovici (2001). We time-step the dynamics starting from rest until an oscillatory steady state is reached. As discussed in Becker and Bercovici (2001), this oscillatory steady-state solution is a forced solution of Eqs. (11)-(16) as the transient free waves of the system are damped. This is why the transverse perturbation is imposed as an applied surface pressure rather than as an initial condition.

The dynamics of Eqs. (11)-(16) depend on properties of the fluid model. These parameters include the nondimensional layer depth and density ratios: $\left(H_{1}^{*}, s\right)$ and the nondimensional viscosities and yield stress parameters: $\left(\Gamma, \Gamma^{\prime}, \Upsilon_{0}, \mathcal{C}\right) . \quad$ As in Becker and Bercovici (2000), we choose the density ratio and upper layer depth as $\left(s, H_{1}^{*}\right)=\left(\frac{1}{1.1}, \frac{1}{3}\right)$, the viscosities and yield stress as $\left(\Gamma, \Gamma^{\prime}, \Upsilon_{0}\right)=(50,1,1.5)$ and consider both the pressuredependent yield stress $(\mathcal{C} \neq 0)$ and constant yield stress $(\mathcal{C}=0)$ bi-viscous rheologies. In the numerical solutions that follow, we choose an empirically determined value for $\mathcal{C}$ so that the yield stress $\Upsilon_{0 p}$ undergoes significant variations, but remains positive.

We solve Eqs. (11)-(16) for the forcing parameters:

$$
\kappa=m \pi, \quad \Omega=\sqrt{H_{1}^{*}} \kappa=\frac{m \pi}{\sqrt{3}}
$$



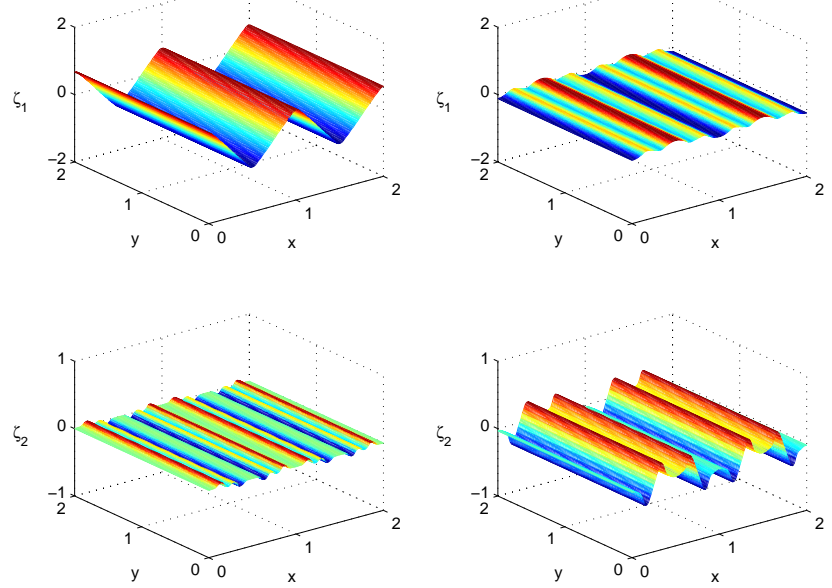

Fig. 2. Purely longitudinal surface pressure forcing. The freesurface (upper panels) and interface displacement (lower panels) at two representative times that satisfy Eqs. (11)-(16) with $\left(s, H_{1}^{*}, \Gamma, \Gamma^{\prime}, \Upsilon_{0}\right)=\left(\frac{1}{1.1}, \frac{1}{3}, 50,1,1.5\right),(\kappa, \Omega)=\left(2 \pi, \frac{2 \pi}{\sqrt{3}}\right), \alpha=0$ and a constant yield stress, $\mathcal{C}=0$ in Eq. (16).

for $m=1,2,3$ so that long-standing waves of wave number $m$ are excited in the upper layer. For reference, we present in Figs. 2 and 3 snapshots at two times over a period of the forcing of the free-surface (upper panels) and interface displacement (lower panels) for zero applied transverse pressure perturbation $(\alpha=0)$ for $m=2$.

We remark that in the runs presented here for $m=2$, we use a spatial resolution of $129 \times 129$ grid points which was sufficient to obtain accurate, converged solutions. For $\alpha=$ 0 , the solutions of Eqs. (11)-(16) remain one-dimensional and the dynamics reduce to those examined in Becker and Bercovici (2000). As mentioned above, the only nonlinearity in the dynamics of Eqs. (11)-(16) is in the rheology, and for a constant yield stress $(\mathcal{C}=0)$ the stress is an odd function of the strain rate for one-dimensional motions. The symmetry of the nonlinear rheology restricts the transfer of energy from the forced wave motion to odd harmonics of the forcing. Figure 2 shows the model response for a bi-viscous lower layer with a constant yield stress $(\mathcal{C}=0)$ that demonstrates (as in Becker and Bercovici, 2000) that the nonlinear lower layer rheology results in the transfer of energy from the directly forced wave to odd spatial harmonics of this forcing. This lower layer spatial pattern oscillates in time with the forcing frequency and hence, is not permanent. For a pressuredependent yield stress, however, the odd symmetry of the stress-strain rate relationship is broken and energy may be transferred to even and odd harmonics of the forcing. As a result, for a pressure-dependent yield stress $(\mathcal{C} \neq 0)$, a spatial pattern in the interface displacement with a significant nonzero time average is generated that has half the wavelength of the forcing (Fig. 3).

We next perturb the dynamics of Eqs. (11)-(16) with the small transverse external surface pressure with wave number
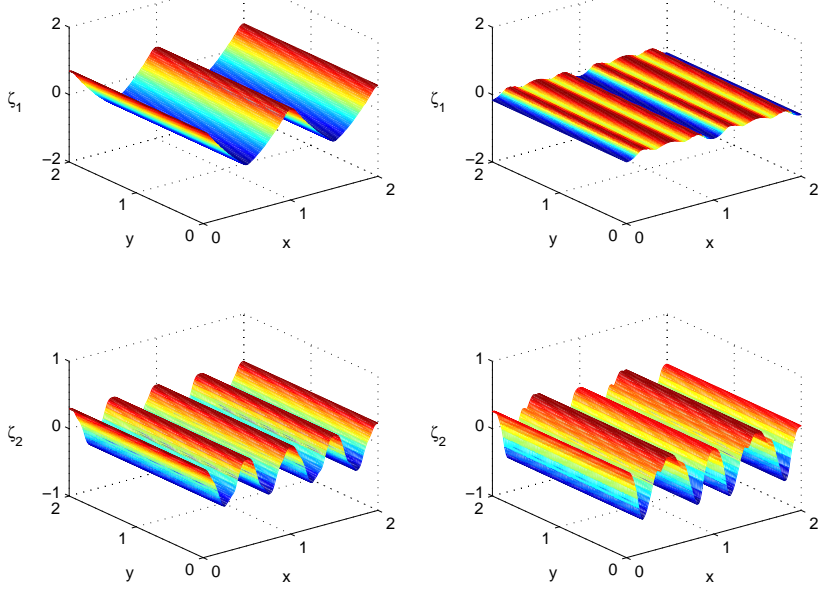

Fig. 3. Same as Fig. 2, but with the pressure-dependent yield stress, $\mathcal{C}=1.2$ in Eq. (16).

and frequency:

$\lambda=n \pi, \quad \Sigma=\sqrt{H_{1}^{*}} \lambda=\frac{n \pi}{\sqrt{3}}$

for $1 \leq n \leq 6,(m=2,3)$ and $1 \leq n \leq 4,(m=1)$. We fix the nondimensional amplitude of these perturbations $\alpha=0.1$, although other values of $\alpha$ were explored. For twodimensional motions, the rheology described by Eqs. (15)(16) for a constant yield stress $(\mathcal{C}=0)$ does provide a pathway for energy to be transferred between longitudinal and transverse motions through the magnitude of the bottom strain-rate $\hat{\dot{\varepsilon}}_{b}^{*}$. We find, however, that for a constant yield stress bi-viscous lower layer, the model response remains quasi-one-dimensional and resembles the one-dimensional solutions presented in Fig. 2 for $\alpha=0$ in all cases examined, except when $\frac{n}{m}=2$. Figures 4 and 5 are analogous to Figs. 2 and 3 and present the free-surface elevation and interface displacement that satisfy Eqs. (9)-(14) for $m=2$, but for the applied transverse pressure perturbation $(\alpha, n)=(0.1,2)$. Comparing Figs. 2 and 4 shows that the transverse pressure perturbation produces only a small forced transverse modulation for the constant yield stress, bi-viscous lower layer and the model results remain quasi-one-dimensional.

For a pressure-dependent yield stress, however, the lower layer response differs dramatically from that obtained for a constant yield stress rheology in some parameter regimes. Specifically, for the numerical experiments conducted when $\frac{n}{m}=\frac{1}{3}, \frac{1}{2}, \frac{2}{3}, 1,2$, the lower layer response is strongly twodimensional with its transverse wavelength always greater than or equal to its longitudinal wavelength. Figure 5 presents the free-surface and interface displacement that satisfy Eqs. (11)-(16) with $n=m=2$ but with $\mathcal{C}=1.2$. Comparing Fig. 4 with Fig. 5 shows that the pressure-dependent yield stress has resulted in the significant amplification of the transverse pressure perturbation. This significant amplification of transverse motions is due to the additional nonlinear coupling between longitudinal and transverse motions in the 

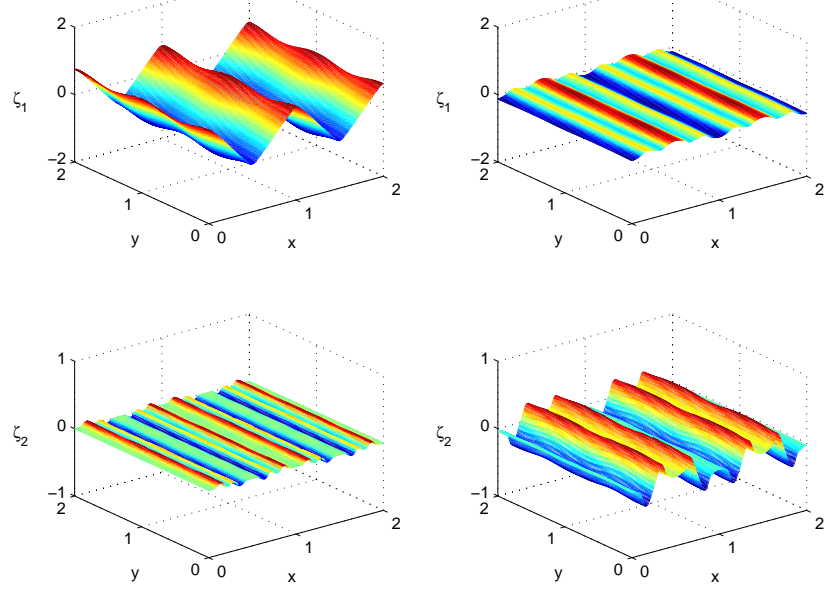

Fig. 4. Effects of a transverse pressure perturbation with $\mathcal{C}=1$ in Eq. (16). Same as Fig. 2, but with the transverse pressure perturbation $(\alpha, \lambda, \Sigma)=\left(0.1,2 \pi, \frac{2 \pi}{\sqrt{3}}\right)$.

rheological model Eqs. (15)-(16) provided by the pressuredependent yield stress.

As mentioned above, the $\frac{n}{m}=2$ solution produces an amplified transverse component for the constant yield stress lower layer. We remark, however, that the amplitude of this transverse component is less than that obtained for the pressure-dependent yield stress. Moreover, for the constant yield stress, the lower layer spatial pattern oscillates with the forcing frequency while for the pressure-dependent yield stress, the lower layer spatial pattern has a significant nonzero time average.

\section{Discussion}

We have considered here the two-dimensional response of a two-layer model of the interaction of long standing waves with a deformable seabed modeled as a bi-viscous fluid. We have shown previously (Becker and Bercovici, 2000) in one dimension that permanent bedforms (i.e. interfacial spatial patterns with a significant non-zero time average over a period of the forcing) do not form on a bi-viscous lower layer with a constant yield stress, but do form on a bi-viscous lower layer with a pressure-dependent yield stress that models the effect that the lower layer (sand bed) is more difficult to move under increased compression. Here, we have sought to determine under what conditions predominantly longitudinal forcing will generate permanent bedforms with significant transverse and longitudinal spatial structures.

Insight into the two-dimensional results presented here may be gained by revisiting the one-dimensional model results. For a bi-viscous lower layer with a constant yield stress, the only nonlinearity in the one-dimensional versions of Eqs. (11)-(16) is in the rheology Eqs. (15)-(16) and the odd symmetry of this nonlinearity results in odd spatial harmonics of the forcing being excited in the lower layer. More-
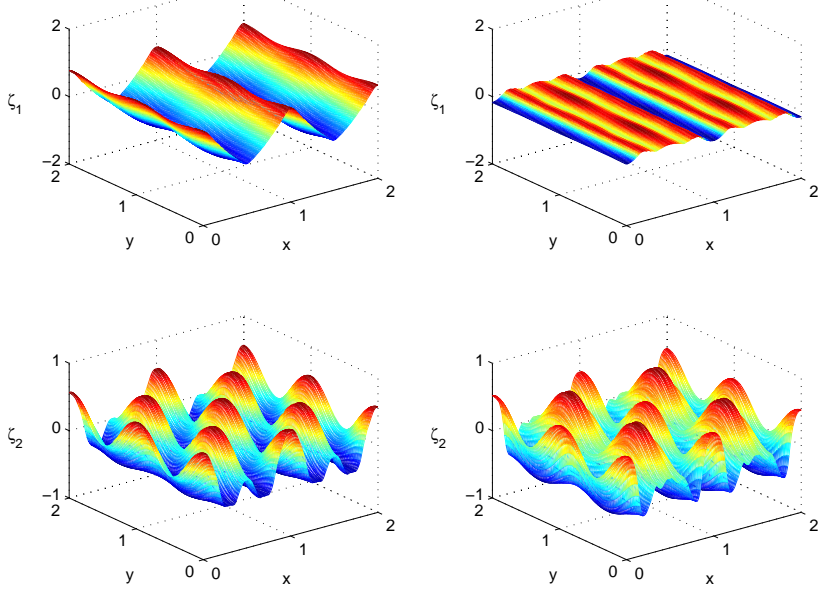

Fig. 5. Effects of a transverse pressure perturbation with $\mathcal{C}=\infty . \in$ in Eq. (16). Same as Fig. 3, but with the transverse pressure perturbation $(\alpha, \lambda, \Sigma)=\left(0.1,2 \pi, \frac{2 \pi}{\sqrt{3}}\right)$.

over, these odd spatial harmonics all oscillate with the forcing frequency. Hence, the lower layer pattern is directly forced in time (i.e. the frequency of the lower layer response is the same as the forcing frequency), but a nonlinear cascade of energy to smaller scales occurs in space (Fig. 2).

For the pressure-dependent yield stress, the odd symmetry of the nonlinear rheology in the one-dimensional dynamics is broken. As a result, energy is transferred to even and odd spatial harmonics of the forcing. While the odd spatial harmonics excited in the lower layer still oscillate with the forcing frequency, the even spatial harmonics of the free surface and interface displacements have a significant time-independent component (Fig. 3). Hence, the history-dependent rheology has resulted in a nonlinear cascade of energy in space and time; with the constant yield stress bi-viscous rheology, significant nonlinear interactions in the lower layer only occurred in space.

In two dimensions, the nonlinear rheology for a constant yield stress remains an odd function of the strain rate in both the longitudinal and transverse directions, and in all cases examined, we find that the interfacial pattern excited oscillates with the forcing frequency (e.g. Fig. 4). The spatial structure of this oscillating pattern typically remains onedimensional (longitudinal), except in the special case when the transverse pressure perturbation has twice the wavelength and frequency of the longitudinal pressure forcing. In this special case, significant coupling between the longitudinal and transverse motions occurs through the magnitude of the bottom strain-rate $\hat{\dot{\varepsilon}}_{b}^{*}$. So while spatial coupling between longitudinal and transverse motions may occur in two dimensions through $\hat{\varepsilon}_{b}^{*}$, the interfacial pattern still is directly forced in time for a constant yield stress and hence, is not permanent.

For a pressure-dependent yield stress, however, we find small initial transverse perturbations are amplified over a 
larger range of perturbation parameters than for a constant yield stress. Moreover, these two-dimensional patterns have a significant non-zero time average (e.g. Fig. 5). Specifically, we find that when the forcing wave number and frequency is greater than or equal to the perturbation wave number and frequency, or in the special case when the perturbation wave number and frequency is twice the forcing wave number and frequency, transverse bedforms are excited with transverse wavelengths greater than or equal to their longitudinal wavelengths. That significant coupling between longitudinal and transverse motions occurs in a larger parameter regime for the pressure-dependent yield stress than the constant yield stress bi-viscous rheology is not surprising since the pressure-dependent yield stress breaks the symmetry of this rheology. With a constant yield-stress, the only coupling between longitudinal and transverse motions occurs through the strain-rate invariant, while for the pressuredependent yield stress, additional coupling between longitudinal and transverse motions is provided by the historydependent rheology. Moreover, as in the one-dimensional case, this history-dependent rheology has resulted in nonlinear temporal interactions leading to the formation of permanent bedforms.

While the present model is highly idealized, it is worthwhile to assess the length scales of the bedforms generated by representative wave conditions. For example, a 10 -s wave in $10 \mathrm{~m}$ of water has a wavelength of approximately $100 \mathrm{~m}$. The longitudinal (cross-shore) wavelength of the one-dimensional interfacial pattern shown in Fig. 4 for a bi-viscous, constant yield stress lower layer is approximately $50 \mathrm{~m}$, while the interfacial pattern for the bi-viscous lower layer with a pressure-dependent yield stress shown in Fig. 5 has a longitudional (cross-shore) wavelength of approximately $50 \mathrm{~m}$ and a transverse (along-shore) wavelength of approximately $100 \mathrm{~m}$. For a unit amplitude wave (crestto-trough), Fig. 6 shows the crest-to-trough height, $\left|\zeta_{2}^{a v}\right|$ of the time-average of the lower layer interfacial pattern

$\zeta_{2}^{a v}=\frac{\Omega}{2 \pi} \int_{t}^{t+\frac{2 \pi}{\Omega}} \zeta_{2}(x, y, t) d t$,

where $\left|\zeta_{2}^{a v}\right|$ is the difference between the maximum and minimum values of $\zeta_{2}^{a v}$, as a function of $\mathcal{C}$, the coefficient of the pressure-dependent yield stress in Eq. (16) for the controlling parameters $\left(s, H_{1}^{*}, \Gamma, \Gamma^{\prime}, \Upsilon_{0}\right)=\left(\frac{1}{1.1}, \frac{1}{3}, 50,1,1.5\right)$, the forcing parameters $(\kappa, \Omega)=\left(2 \pi, \frac{2 \pi}{\sqrt{3}}\right)$ and perturbation parameters $(\alpha, \lambda, \Sigma)=\left(0.1,2 \pi, \frac{2 \pi}{\sqrt{3}}\right)$. For $\mathcal{C}=0$, the timeaverage of the interface displacement is zero (see also Fig. 4). As $\mathcal{C}$ is increased, the two-dimensional interfacial pattern of Fig. 5 (lower panels) develops with a time-averaged crest-totrough amplitude $\left|\zeta_{2}^{a v}\right|$ that increases with increasing $\mathcal{C}$. We emphasize that while both the predicted amplitudes of the bedforms and their transverse wavelength depend upon the controlling and forcing parameters, for all cases examined the longitudinal wavelength of the interfacial pattern is half that of the forcing.

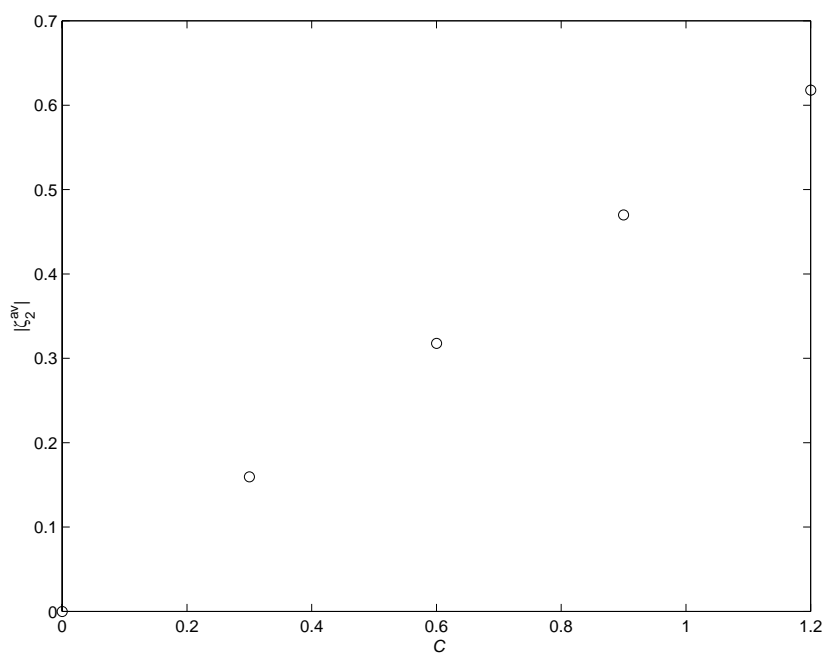

Fig. 6. Dependence of the time-averaged interface displacement $\left|\zeta_{2}^{a v}\right|$ as a function of $\mathcal{C}$, the parameter controlling the pressure dependence of the yield stress in Eq. (16) for a freesurface displacement of unit amplitude and $\left(s, H_{1}^{*}, \Gamma, \Gamma^{\prime}, \Upsilon_{0}\right)=$ $\left(\frac{1}{1.1}, \frac{1}{3}, 50,1,1.5\right)$, the forcing parameters $(\kappa, \Omega)=\left(2 \pi, \frac{2 \pi}{\sqrt{3}}\right)$ and perturbation parameters $(\alpha, \lambda, \Sigma)=\left(0.1,2 \pi, \frac{2 \pi}{\sqrt{3}}\right)$.

We conclude by noting that theoretical models of the coupled interaction of waves with a deformable lower layer are useful in isolating the effects of specific dynamical processes on this interaction. For example, we have shown that a simple constant yield stress bi-viscous lower layer rheology does not allow for the formation of two-dimensional permanent bedforms by standing-wave forcing. By examining a slightly more realistic (although still highly idealized) rheology that incorporates a packing condition through a pressure-dependent yield stress, however, we find that twodimensional permanent lower layer patterns are excited in some parameter regimes.

Acknowledgements. This paper is funded in part by a grant/cooperative agreement (NA86RG0041) from the National Oceanic and Atmospheric Administration, project \# R/EP-9, which is sponsored by the University of Hawaii Sea Grant College Program, SOEST, under Institutional Grant No. NA86RG-0041 from NOAA office of Sea Grant, Department of Commerce. The views expressed herein are those of the authors and do not necessarily reflect the views of NOAA or any of its subagencies. UNIHI-SEAGRANT-JC-01-01. JB also is supported by NSF-OCE-9633057. This is SOEST contribution no. 5858.

\section{References}

Becker, J. M. and Bercovici, D.: Permanent bedforms in a theoretical model of wave-sea-bed interactions, Nonlin. Proc. Geophys., 7, 31-35, 2000.

Becker, J. M. and Bercovici, D.: Pattern formation on the interface of a two-layer fluid: Bi-viscous lower layer, Wave Motion (34), 431-452, 2001. 
Blondeaux, P.: Sand ripples under sea waves Part 1. Ripple formation, J. Fluid Mech., 218, 1-17, 1990.

Boczar-Karakiewicz, B., Forbes, D. L., and Drapeau, G.: Nearshore bar development in the southern Gulf of St. Lawrence, J. Waterways, Port, Coastal and Ocean Eng., ASCE, 121, 49-60, 1995.

Carter, T. G., Liu, P. L-F., and Mei, C. C.: Mass transport by waves and offshore sand bedforms, J. Waterways, Harbors, and Coastal Eng. Div., ASCE, 99 (WW2), 165-184, 1973.

Deigaard, R. N. Dronen, Fredsoe, J., Hjelmager Jensen, J., and Jorgensen, M. P.: A morphological stability analysis for a long straight barred coast, Coastal Eng., 36, 171-195, 1999.

Falques, A., Montoto, A., and Iranzo, V.: Bed-flow instability of the longshore current, Continental Shelf Res,, 16 (15), 1927-1964, 1996.

Holman, R. A.: The interaction of waves, currents and nearshore bathymetry, in:Proceedings Aha Hulikoa Hawaiian Winter Workshop, (Eds) Muller, P. and Henderson, D., 257-262, SOEST Special Publication, 1995.

Holman, R. A. and Bowen, A. J.: Bars, bumps, and holes: models for the generation of complex beach topography, J. Geophys. Res., 87 (C1), 457-468, 1982.

Komar, P. D.: Beach Processes and Sedimentation, 544, Prentice Hall, New Jersey, 1998.

Liu, K.-F. and Mei, C. C.: Long waves in shallow water over a layer of Bingham-plastic fluid-mud-I. Physical Aspects, Int. J. Engng. Sci., 31(1), 125-144, 1993a.
Liu, K.-F. and Mei, C. C.: Long waves in shallow water over a layer of Bingham-plastic fluid-mud-II. Mathematical derivation of long wave equations, Int. J. Engng. Sci., 31(1), 145-155, 1993b.

Liu, K.-F. and Mei, C. C.: Slow spreading of a sheet of Bingham fluid on an inclined plane, J. Fluid Mech., 207, 505-529, 1989.

$\mathrm{Ng}$, C.-O. and Mei, C. C.: Roll waves on a shallow layer of mud modeled as a power-law fluid, J. Fluid Mech., 263, 151-183, 1994.

Plant, N. G., Holman, R. A., Freilich, M. H., and Birkemeier, W. A.: A simple model for interannual sandbar behaviour, J. Geophys. Res., 104 (C7), 15, 755-776, 1999.

Roelvink, J. A. and Broker, I., Cross-shore profile models, Coastal Eng., 21, 163-191, 1993.

Thornton, E., Dalrymple, T., Drake, T., Gallagher, E., Guza, B., Hay, A., Holman, R., Kaihatu, J., Lippmann, T., and OzkanHaller, T.: Nearshore research, Report of the Nearshore Research Workshop, St. Petersburg, Florida, 14-16 September 1998 http://www.oc.nps.navy.mil/ thornton/

Vittori, G. and Blondeaux, P.: Sand ripples under sea waves Part 2. Finite-amplitude development, J. Fluid Mech., 218, 19-39, 1990.

Vittori, G. and Blondeaux, P.: Sand ripples under sea waves Part 3. Brick-pattern ripples formation, J. Fluid Mech., 239, 23-45, 1992.

Werner, B. T. and Fink, T. M.: Beach cusps as self-organized patterns, Science, 260, 968-971, 1993. 